THE ASTROPHYSICAL JOURNAL, 513:207-214, 1999 March 1

(c) 1999. The American Astronomical Society. All rights reserved. Printed in U.S.A.

\title{
HIGH SPATIAL RESOLUTION IMAGING OF ARP 220 FROM 3 TO 25 MICRONS $^{1}$
}

\author{
B. T. Soifer, ${ }^{2}$ G. Neugebauer,${ }^{2}$ K. Matthews,${ }^{2}$ E. E. Becklin, ${ }^{2}$ M. Ressler, ${ }^{3}$ M. W. Werner, ${ }^{3}$ \\ A. J. WeINBERGER, ${ }^{2,4}$ AND E. EGAMI ${ }^{2}$ \\ Received 1998 July 27; accepted 1998 October 6
}

\begin{abstract}
Images of Arp 220 from 3.45 to $24.5 \mu \mathrm{m}$ with 0 ".5 resolution are presented that clearly separate the nucleus into at least two components. The western component is about 3 times more luminous than the eastern component, but the silicate absorption in the fainter, eastern component is roughly $50 \%$ greater than the absorption in the western component. Each component is marginally resolved. The two components seen at $24.5 \mu \mathrm{m}$ are identified with the two radio components. The western source most likely coincides with the high-extinction disk previously suggested to exist in Arp 220, while the eastern nucleus is identified with a faint, highly reddened source seen in HST $2.2 \mu \mathrm{m}$ NICMOS images. The two nuclei together account for essentially all of the measured $24.5 \mu \mathrm{m}$ flux density. Two models are presented, both of which fit the observations. In one the majority of the total luminosity is produced in an extended star formation region, and in the other most of the luminosity is produced in the compact but extincted regions associated with the two nuclei seen at $24.5 \mu \mathrm{m}$. In both pictures, substantial luminosity at $100 \mu \mathrm{m}$ emerges from a component having a diameter of $2^{\prime \prime}-3^{\prime \prime}(\sim 1 \mathrm{kpc})$.
\end{abstract}

Subject headings: galaxies: individual (Arp 220) — galaxies: nuclei - galaxies: structure infrared: galaxies

\section{INTRODUCTION}

Since its identification as a highly luminous galaxy in the infrared (Soifer et al. 1984), Arp 220 (IC 4553/4) has undergone extensive observational studies with the recognition that it is the closest of the ultraluminous infrared galaxies (Sanders et al. 1988). Its bolometric luminosity of $1.2 \times 10^{12}$ $L_{\odot}$ is in the range of quasar luminosities, but most recently Arp 220 has been cited as a prototype for the appearance of forming galaxies at high redshifts (van der Werf 1998).

The most striking property of Arp 220 is the very strong infrared emission that rises rapidly from $\sim 0.5 \mathrm{Jy}$ at $12 \mu \mathrm{m}$, to $\sim 10 \mathrm{Jy}$ at $25 \mu \mathrm{m}$, and to a peak flux density of $\sim 100 \mathrm{Jy}$ at $60 \mu \mathrm{m}$ (Soifer et al. 1984; Soifer et al. 1987). This emission is generally attributed to thermal emission by dust heated by an underlying luminosity source; the dust emission at these wavelengths accounts for more than $98 \%$ of the bolometric luminosity of the source. Wynn-Williams \& Becklin (1993) showed that the size of the source at $32 \mu \mathrm{m}$ was less than $2^{\prime \prime}$ and that the source was centered on the optically obscured nucleus of the galaxy. This requires the luminosity source to be either a dense starburst, a heavily dustenshrouded active galactic nucleus (AGN), or some combination of these sources.

Recent observational studies of Arp 220 have revealed new details of the inner few arcseconds or $1 \mathrm{kpc}$ in this system. Graham et al. (1990) resolved the nuclear region at $2.2 \mu \mathrm{m}$ into two nuclei separated by 0.95 and suggested that

\footnotetext{
${ }^{1}$ Based in part on observations obtained at the W. M. Keck Observatory, which is operated jointly by the California Institute of Technology and the University of California.

2 Palomar Observatory, California Institute of Technology, 320-47, Pasadena, CA 91125; bts@mop.caltech.edu, gxn@ caltech.edu, kym@caltech.edu, alycia@astro.ucla.edu, egami@mop.caltech.edu.

3 Jet Propulsion Lab, 169-506, 4800 Oak Grove Drive, Pasadena, CA 91109; mww@ipac.caltech.edu, ressler@cougar.jpl.nasa.gov.

${ }^{4}$ Department of Physics and Astronomy, University of California Los Angeles, 156205 Los Angeles, CA 90095; becklin@astro.ucla.edu.
}

they were remnants of merging galaxy nuclei that were inferred from the large tidal tails seen in optical imagery (Arp 1966; Toomre \& Toomre 1972). Larkin et al. (1995) established from near-infrared spectroscopy of the nuclei that they appeared to be orbiting and placed a lower limit of $1.5 \times 10^{9} M_{\odot}$ on the total mass of the orbiting nuclei. Finally, Scoville et al. (1998) have presented NICMOS data from 1.1 to $2.2 \mu \mathrm{m}$ that resolve the entire region into several sources.

Condon et al. (1991) and Baan \& Haschick (1995) separated the nucleus into two peaks in the radio continuum, and Scoville et al. (1998) matched the radio peaks with two peaks seen in the $2.2 \mu \mathrm{m}$ NICMOS images. Smith et al. (1998) have reported VLBI observations of Arp 220 at 18 $\mathrm{cm}$ with resolution of $\sim 5$ mas that resolve the continuum of the separate nuclei into discrete point sources that they interpret as supernova remnants within the nuclear starbursts. Downes \& Solomon (1998) and Sakamoto et al. (1998) have reported new millimeter-wave interferometry with $\sim 0$ ".5 resolution that has resolved gaseous disks associated with each of the nuclei.

Previous midinfrared imaging of Arp 220 has been reported by Keto et al. (1992) and Miles et al. (1996). Keto et al. (1992) reported observations in a broad band from 8 to $13 \mu \mathrm{m}$ that constrain the nuclear region to be less than 1 1.3 $\times 0$ 0.9. Miles et al. (1996) reported observations at 8.8 and $12.5 \mu \mathrm{m}$ with resolutions of 1.0 and $0{ }^{\prime \prime} 7$ that showed that the nuclear region at these wavelengths is basically a double source elongated mostly east-west, with the western source slightly resolved and having 1.6 and 3.3 times the flux density of the eastern source at 8.8 and $12.5 \mu \mathrm{m}$, respectively.

In this paper we report infrared imaging at wavelengths from 3.45 to $24.5 \mu \mathrm{m}$ with angular resolution of 0.3 to 0.6 . These data directly probe the distribution of thermally emitting dust in the nuclear region and trace the luminosity in this source at wavelengths approaching the peak of the output for this system. Throughout this paper, we will 
assume a Hubble constant of $75 \mathrm{~km} \mathrm{~s}^{-1} \mathrm{Mpc}^{-1}$. At a redshift of $5400 \mathrm{~km} \mathrm{~s}^{-1}$, Arp 220 is thus at a distance of $72 \mathrm{Mpc}$ and $1^{\prime \prime} \approx 350 \mathrm{pc}$.

\section{OBSERVATIONS AND DATA REDUCTION}

The observations reported here were obtained primarily with the MIRLIN midinfrared camera (Ressler et al. 1994) at the $\mathrm{f} / 40$ bent Cassegrain visitor port of the Keck II Telescope of the W. M. Keck Observatory. The camera used a $128 \times 128 \mathrm{Si}$ :As array and was configured to have a plate scale of 0 .'138 pixel $^{-1}$ for a total field of view of $17^{\prime \prime} \times 17^{\prime \prime}$. Observations were obtained in eight infrared bands from 7.9 to $24.5 \mu \mathrm{m}$. In the $10 \mu \mathrm{m}$ atmospheric window, observations were made with six different intermediate bandpass filters in order to sample the spectral features of dust emission in this window. Two additional filters covered the ends of the $20 \mu \mathrm{m}$ atmospheric window and were selected to provide wavelength coverage reaching the longest wavelengths easily observed by ground-based telescopes short of submillimeter wavelengths. The central wavelengths and bandpasses of the filters are listed in Table 1.

The MIRLIN observations presented here were made on the night of 1998 March 18 UT. At each wavelength the observing procedure was the same. A chopping secondary with a square wave chop, an amplitude of $6^{\prime \prime}$ in the northsouth direction, and a frequency of approximately $4 \mathrm{~Hz}$ was employed for fast beam switching. The frames at each chop position were co-added in hardware, resulting in two images. After an interval of approximately a minute, the telescope was nodded east-west by 6 ", and a second set of two images was obtained in order to cancel residuals in the sky and subtract telescope emission. This procedure was repeated a number of times, giving a total time on the source between 2 and 10 minutes, depending on the strength of the source. A nearby star, $\beta$ Her, was observed just before the nucleus of Arp 220 was observed in order to determine the point-spread function (PSF). As with all objects measured throughout the night, $\beta$ Her was observed sequentially at all the relevant wavelengths, i.e., observations of $\beta$ Her at all wavelengths were completed before the nucleus of Arp 220 was observed. The FWHM of $\beta$ Her, as well as that of five other stars measured throughout the night, was $\lesssim 0$ ".2 greater than the diffraction limit at the shorter observing wavelengths but was diffraction limited, 0 ."62 at $24.5 \mu \mathrm{m}$. Because the measured image size of the PSF stars varied significantly over the night, we do not feel confident in attributing differences $\lesssim 0$ ". 2 between a mea-

TABLE 1

OBSERVING BANDS

\begin{tabular}{ccc}
\hline \hline $\begin{array}{c}\text { Central Wavelength } \\
(\mu \mathrm{m})\end{array}$ & $\begin{array}{c}\text { Half-Power Width } \\
(\mu \mathrm{m})\end{array}$ & $\begin{array}{c}\text { Flux Density } \\
\text { at 0.0 mag }(\mathrm{Jy})\end{array}$ \\
\hline $3.45 \ldots \ldots \ldots \ldots \ldots \ldots$. & 0.24 & 292 \\
$7.9 \ldots \ldots \ldots \ldots \ldots \ldots \ldots$ & 0.76 & 63.3 \\
$8.8 \ldots \ldots \ldots \ldots \ldots \ldots \ldots$ & 0.87 & 51.5 \\
$9.7 \ldots \ldots \ldots \ldots \ldots \ldots \ldots$ & 0.93 & 42.7 \\
$10.3 \ldots \ldots \ldots \ldots \ldots \ldots \ldots$ & 1.01 & 38.0 \\
$11.7 \ldots \ldots \ldots \ldots \ldots \ldots \ldots$ & 1.11 & 29.7 \\
$12.5 \ldots \ldots \ldots \ldots \ldots \ldots \ldots$ & 1.16 & 26.2 \\
$17.9 \ldots \ldots \ldots \ldots \ldots \ldots \ldots$ & 2.00 & 7.0 \\
$24.5 \ldots \ldots \ldots \ldots \ldots \ldots$ & 0.76 & 7.0 \\
\hline
\end{tabular}

sured source size and the PSF size to an intrinsic source size, except possibly at wavelengths of $24.5 \mu \mathrm{m}$.

The data were reduced by differencing the two images obtained within the chop pairs at each nod location and then coadding the resulting four positive images, with their positions appropriately adjusted to a common location, to yield a positive image centered in a field approximately $6^{\prime \prime} \times 6^{\prime \prime}$.

The entire night was photometric, and the sensitivity was based on measurements of $\alpha$ Tau made at the beginning of the evening. The photometric measurements of $\alpha$ Tau and of the five stars made at different wavelengths throughout the night are consistent to $\lesssim 10 \%$ with previous groundbased measurements and the color-corrected IRAS Point Source Catalog flux densities. The flux density corresponding to $0.0 \mathrm{mag}$ was taken to follow the prescription given in the IRAS Explanatory Supplement (1988) and is listed in Table 1.

In addition to observations at wavelengths $\lambda \geq 7.9 \mu \mathrm{m}$, observations at high spatial resolution at $3.45 \mu \mathrm{m}(L)$ were obtained of the nucleus of Arp 220 using the Cassegrain infrared camera on the 200 inch $(5.08 \mathrm{~m})$ Hale Telescope of Palomar Observatory on 1997 May 20 in nonphotometric conditions. Thirteen pairs of observations, each with $70 \mathrm{~s}$ of integration (1000 co-added $0.07 \mathrm{~s}$ frames) on the galaxy and on nearby sky, were obtained with a $64 \times 64$ pixel subarray of the InSb array camera. The pixel scale of the camera was 0 ". 125 pixel $^{-1}$, and hence the field of view was $8^{\prime \prime} \times 8^{\prime \prime}$. Each image of the galaxy was sky-subtracted and flat-fielded. Residual sky levels were estimated for each image and subtracted. Bad pixels were corrected by interpolation. The 13 resulting images were registered using the recorded positions of the offset guider and averaged. In order to calibrate the final image photometrically, further $3.45 \mu \mathrm{m}$ images were obtained of the galaxy and of a photometric standard on 1998 June 5, a photometrically good night, and processed in the same manner as described above.

\section{RESULTS}

The nuclear region of Arp 220 was detected at all the wavelengths where it was observed. The photometry of the nuclear region within a $4^{\prime \prime}$ diameter beam and within smaller beams (described below) within the nuclear region is presented in Table 2. The total detected flux density of 16 $\mathrm{mJy}$ at $3.45 \mu \mathrm{m}$ is consistent with the flux density of $22 \mathrm{mJy}$ in a $5^{\prime \prime}$ diameter beam at $3.7 \mu \mathrm{m}$ reported by Sanders et al. (1988). At $24.5 \mu \mathrm{m}$ the flux density reported here of $9.8 \mathrm{Jy}$ in a 4" diameter beam is likewise consistent with the flux density of $8.4 \mathrm{Jy}$ measured by Wynn-Williams \& Becklin (1993) at $25 \mu \mathrm{m}$ with a 5.7 diameter beam and with the color-corrected flux density of $10 \mathrm{Jy}$ obtained by IRAS at 25 $\mu \mathrm{m}$ in a rectangular beam $\sim 1^{\prime} \times 5^{\prime}$.

A montage of images at all observed wavelengths is presented in Figure 1a. As a comparison, contour maps of images of the star $\alpha$ Tau, taken before the images of Arp 220 were obtained, are shown in Figure $1 b$. In Figure $1 a$ the centroid of the western nucleus has been set in the same position in each frame on the assumption that the centroid of this source is spatially coincident at all wavelengths. As noted by Scoville et al. (1998), this is a dubious assumption at the shorter wavelengths owing to the presence of heavy extinction in the nuclear region. To facilitate a comparison of the morphologies at all wavelengths, the location of the peak of the eastern component in the image obtained at 
TABLE 2

Photometric Results

\begin{tabular}{|c|c|c|c|c|c|}
\hline \multirow[b]{2}{*}{$\begin{array}{l}\text { CENTRAL WAVELENGTH } \\
(\mu \mathrm{m})\end{array}$} & \multicolumn{3}{|c|}{ FLUX DENSITY $^{a}$} & \multicolumn{2}{|c|}{ FWHM } \\
\hline & $\begin{array}{l}\text { Total }^{\mathrm{b}} \\
(\mathrm{mJy})\end{array}$ & $\begin{array}{l}\text { West }^{\mathrm{e}} \\
(\mathrm{mJy})\end{array}$ & $\begin{array}{l}\text { East }^{\mathrm{d}} \\
(\mathrm{mJy})\end{array}$ & $\begin{array}{l}\text { West }^{\mathrm{e}} \\
(\operatorname{arcsec})\end{array}$ & $\begin{array}{c}\mathrm{PSF}^{\mathrm{f}} \\
(\operatorname{arcsec})\end{array}$ \\
\hline $3.45 \ldots \ldots$. & $16 \pm 2$ & $5.0 \pm 0.5$ & $3.6 \pm 0.3$ & 0.69 & 0.42 \\
\hline $7.9 \ldots \ldots$ & $521 \pm 15$ & $304 \pm 3$ & $159 \pm 4$ & 0.45 & 0.38 \\
\hline $8.8 \ldots \ldots \ldots \ldots \ldots \ldots \ldots$ & $155 \pm 6$ & $103 \pm 1$ & $58 \pm 3$ & 0.42 & 0.35 \\
\hline $9.7 \ldots \ldots \ldots \ldots \ldots \ldots \ldots$ & $124 \pm 14$ & $91 \pm 4$ & $31 \pm 4$ & 0.52 & 0.38 \\
\hline $10.3 \ldots \ldots$ & $59 \pm 10$ & $52 \pm 2$ & $12 \pm 4$ & 0.48 & 0.44 \\
\hline $11.7 \ldots$ & $175 \pm 8$ & $121 \pm 2$ & $56 \pm 2$ & 0.42 & 0.39 \\
\hline $12.5 \ldots \ldots \ldots \ldots \ldots \ldots \ldots$ & $404 \pm 12$ & $269 \pm 3$ & $102 \pm 2$ & 0.48 & 0.40 \\
\hline $17.9 \ldots \ldots \ldots \ldots \ldots \ldots \ldots$ & $1170 \pm 50$ & $1030 \pm 2$ & $228 \pm 14$ & 0.52 & 0.56 \\
\hline $24.5 \ldots \ldots \ldots \ldots \ldots \ldots \ldots$ & $9800 \pm 200$ & $7480 \pm 100$ & $2231 \pm 45$ & 0.73 & 0.62 \\
\hline
\end{tabular}

${ }^{a}$ The uncertainties are statistical; see the text.

b Flux density in a 4 " diameter circular beam centered on the western source.

c Flux density in a 0 ". 8 diameter circular beam centered on the western source.

d Flux density in a $0.7 \times 1$ ".8 rectangular beam shown in Fig. $1 a$.

${ }^{\mathrm{e}} \mathrm{FWHM}$ of the western source.

${ }^{\mathrm{f}}$ FWHM of the PSF, $\beta$ Her.

$24.5 \mu \mathrm{m}$ has been marked by a plus sign at the same relative location on all the images. Since these observations were made using the chopping secondary and nodding, the images are not sensitive to structure on spatial scales $\gtrsim 5^{\prime \prime}$.

At all wavelengths the western nucleus is detected strongly. An eastern nucleus is also present except at 9.7 and 10.3 $\mu \mathrm{m}$, where the signal-to-noise ratios are not adequate to locate it with confidence. While the apparent peak of the eastern emission is at the same location at 7.9, 8.8, and 17.9 $\mu \mathrm{m}$, the peak brightness lies to the northeast of the $7.9 \mu \mathrm{m}$ centroid at 3.45 and $11.7 \mu \mathrm{m}$ but appears to be to the south by about 0.2 at 12.5 and $24.5 \mu \mathrm{m}$. At $3.45 \mu \mathrm{m}$ the eastern nucleus seems to be at the same location as the dominant eastern source seen by Scoville et al. (1998) at 1.1-2.2 $\mu \mathrm{m}$, while at the longer wavelengths, the dominant eastern component apparently agrees in location more with a faint source seen at $2.2 \mu \mathrm{m}$ to the southeast by Scoville et al. (1998) and with the center of the radio continuum sources seen by Smith et al. (1998). The 3.45, 7.9, and $8.8 \mu \mathrm{m}$ images show that the eastern peak is itself elongated at a position angle of $\sim 15^{\circ}$; this is quite similar to the structure revealed in the NICMOS images (Scoville et al. 1998). This structure might also be present at 11.7 and $12.5 \mu \mathrm{m}$, but is not clearly evident at longer wavelengths. At $24.5 \mu \mathrm{m}$ the two nuclei are separated by $0.94 \pm 0.05$ at a position angle of $102^{\circ} \pm 5^{\circ}$, in excellent agreement with the separation of the mean of the radio peaks given by Smith et al. of 0".94 \pm 0 0.01 at a position angle of $102^{\circ} \pm 3^{\circ}$.

The complicated structure of the nuclear region makes accurate photometry of its constituents impossible. In addition to the $4^{\prime \prime}$ diameter circular beams, photometry was obtained on two smaller areas within the nuclear region-a circular beam of 0 ."8 diameter centered on the western nucleus and a rectangular beam $0.7 \times 11^{\prime \prime} .8$ located as outlined in Figure 1a. For the smaller beams, whose dimensions are in some cases comparable to the size of the sources, the sensitivity was adjusted by applying a similar beam to the star observed just prior to the Arp 220 observations. An additional correction of $33 \%$ was applied to the 0 ".8 diameter photometry of the western source at $24.5 \mu \mathrm{m}$ and, on the assumption the source is the same size at 17.9 $\mu \mathrm{m}$ as at $24.5 \mu \mathrm{m}$, at $17.9 \mu \mathrm{m}$ to account for the fact that the source was extended while the PSF was not. The accuracy of this correction depends on the observation that the 24.5 $\mu \mathrm{m}$ observations throughout the night were diffractionlimited despite the variability of the seeing at shorter wavelengths. The uncertainties given in Table 2 are dominated by photon statistics and do not include systematic uncertainties. The calibration of the magnitude of the standard star and the zero points are probably uncertain at the $10 \%$ level. The uncertainties in the small beam relative to each other and to the large-beam measurements are in the $10 \%-20 \%$ range, owing primarily to the uncertainties in the detected source sizes.

The western source appears to be marginally resolved. The measured FWHM of the western nucleus and the FWHM of a star observed at each wavelength just prior to the observations of Arp 220 are given in Table 2 and are shown by the clear and hatched concentric circles, respectively, in each panel of Figure 1a. In all cases except 17.9 $\mu \mathrm{m}$, the FWHM of the western nucleus exceeds that of the PSF star, indicating that the western source is resolved. The $17.9 \mu \mathrm{m}$ measurement, where the source FWHM is smaller than that of the PSF, apparently reflects a fluctuation in the seeing. The discrepancy can perhaps be taken as an indication of the uncertainties in any size determination, which are presumably caused by seeing variations.

Figure $1 a$ suggests that a low level of extended emission extends beyond the two nuclei and has an overall extent of $\approx 2^{\prime \prime}-3^{\prime \prime}(700-1000 \mathrm{pc})$ at most wavelengths. The photometric measurements indicate that this emission is small. A comparison of the various measurements given in Table 2 indicates that at most $10 \%$ of the emission at wavelengths longer than $3.45 \mu \mathrm{m}$ is in this extended component, while a comparison between the emission at $24.5 \mu \mathrm{m}$ and the $25 \mu \mathrm{m}$ IRAS measurements indicates that to within the uncertainties of the calibration, the emission within the $1^{\prime} \times 5^{\prime}$ IRAS beam is contained in the source area shown in Figure $1 a$.

Figure 2 shows the spectral energy distribution of the entire source within the $4^{\prime \prime}$ diameter beam as well as of the individual components within the two smaller beams described above. The spectral energy distributions show deep minima near $10 \mu \mathrm{m}$ that presumably indicate strong 


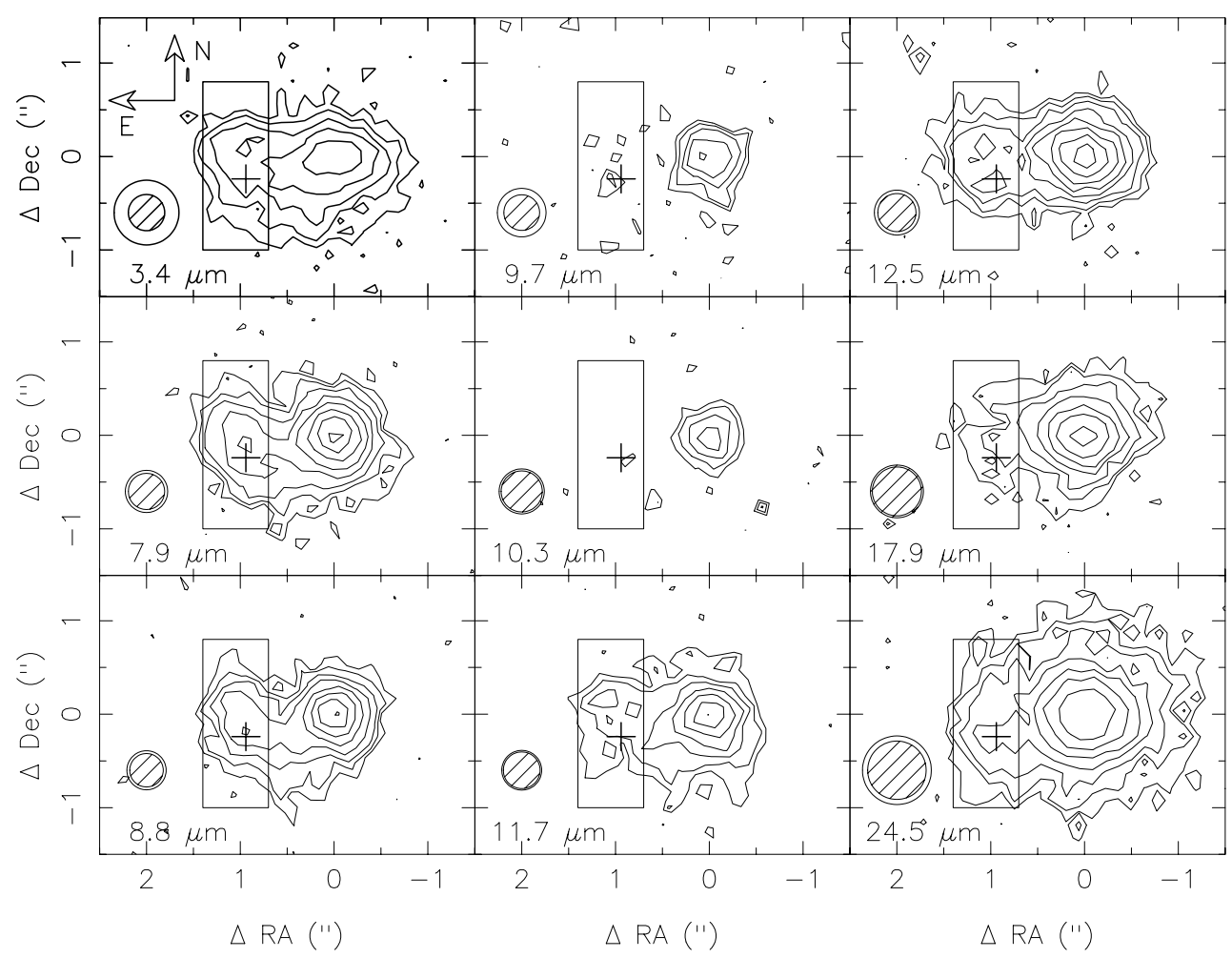

FIG. $1 a$

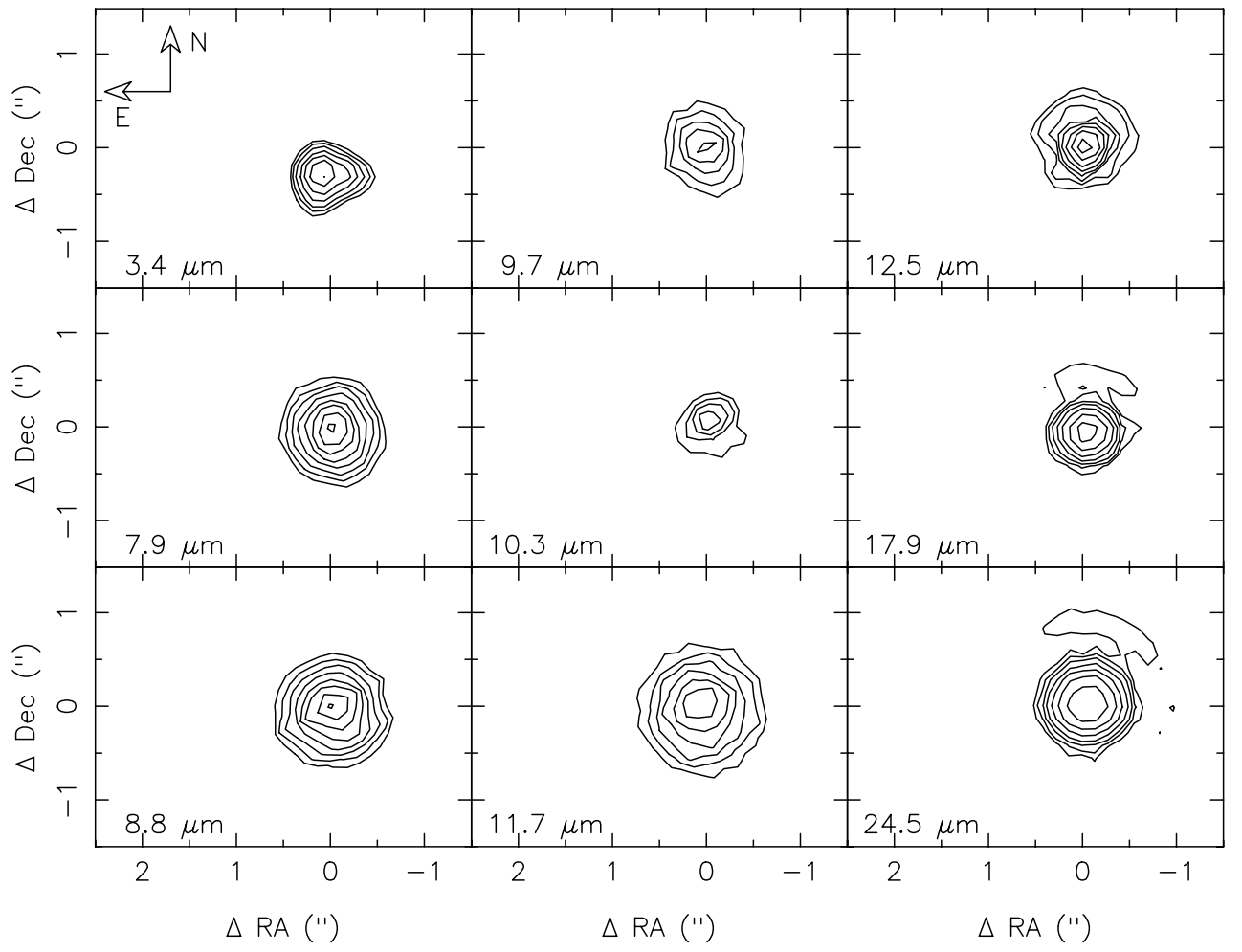

FIG. $1 b$

Fig. 1.- (a) Contour maps are shown of Arp 220 as observed at the nine different wavelengths indicated. North is up and east is to the left in all frames. The level of the lowest contour has been arbitrarily set at the mode of the sky adjoining the galaxy plus 3 times the rms noise of that sky. In order to emphasize the low-level features of the image, the lowest contour interval has been set to the rms noise level of the sky; subsequent intervals increase by factors of 1.5. At each wavelength the FWHM of the star $\beta$ Her taken just before the observations of Arp 220 is indicated in the hatched circle, while the FWHM of the western nucleus is indicated by the clear circle. The plus sign is located at the same place with respect to the western nucleus in all the images, at the peak of the eastern nucleus at $24.5 \mu \mathrm{m}$. The rectangles outline the area used for photometry of the eastern source. (b) Contour maps of the star $\alpha$ Tau taken before the Arp 220 observations are shown at the same scale as $(a)$. The contours have been selected to correspond to those of the corresponding frames in (a) when the extreme difference in brightness is taken into account. The bar over the wavelength identification corresponds to a $1.44 *$ wavelength/telescope diameter. 


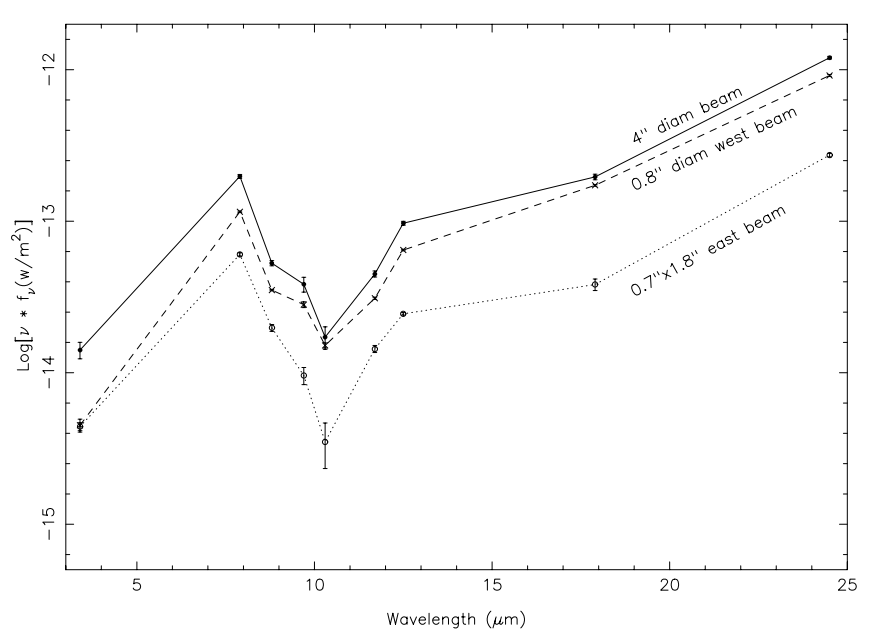

FIG. 2.-The spectral energy distribution of a 4 " diameter beam centered on the western nucleus is shown together with those of a 0.8 diameter beam centered on the western nucleus and a $0.7 \times 11^{\prime \prime} .8$ rectangular beam at the position outlined in Fig. 1 $a$. The flux densities have been corrected for the finite beam size as described in the text.

absorption (Smith, Aitken, \& Roche 1989). Other than the depth of the absorption, the overall spectral energy distributions of the two locations are quite similar within the uncertainties.

\section{DISCUSSION}

The appearance of the nucleus of Arp 220 in the midinfrared is complex because of the interplay of background light, embedded energy sources, and wavelength-dependent emission and absorption by dust. This complexity is already clearly apparent in the 1.1, 1.6, and $2.2 \mu \mathrm{m}$ NICMOS images of Scoville et al. (1998), which show the effects of large and highly variable extinction on the background starlight emission from the nuclear environments. The midinfrared data and, in particular, the $24.5 \mu \mathrm{m}$ data are, however, important in developing a picture of Arp 220, because a significant fraction of the total luminosity of the system is emerging at these wavelengths. Further, the strong absorption, presumably due to silicates (see, e.g., Smith et al. 1989), that is apparent in the spectral energy distributions of both nuclei implies that there is a significant column of intervening cooler material in the line of sight to those regions.

The relative locations of the western and southeastern peaks at $24.5 \mu \mathrm{m}$ are the same to within the uncertainties as the relative locations of the western clump of unresolved radio sources to the southeastern clump (Smith et al. 1998). Thus we associate the peaks seen at $24.5 \mu \mathrm{m}$ with the radio peaks seen by Condon et al. (1991), Baan \& Haschick (1995), and Smith et al. (1998). With this assumption, the southeastern peak is located within $00^{\prime \prime} 1$ of the area associated with the largest dust absorption as inferred by Scoville et al. (1998). Based on the agreement between the separation and position angles of the eastern and western nuclei at 24.5 $\mu \mathrm{m}$ and $1.3 \mathrm{~mm}$ (Downes \& Solomon 1998; Sakamoto et al. 1998), we also assume that the peaks at these wavelengths are spatially coincident. The agreement between the distribution of $24.5 \mu \mathrm{m}$ flux and the continuum at $1.3 \mathrm{~mm}$ is important, since the $1.3 \mathrm{~mm}$ emission is presumably opti- cally thin and thus traces the total dust column density in the nuclear regions in this system.

We attribute the strong dip near $10 \mu \mathrm{m}$ (Fig. 2) to absorption by silicates. The eastern region has a significantly deeper absorption than the western source around $10 \mu \mathrm{m}$. The observations at $17.9 \mu \mathrm{m}$ show a dip, a further signature of silicates, in both regions, but especially in the eastern source. The ground-based $10 \mu \mathrm{m}$ spectra of Smith et al. (1989) as well as the Infrared Space Observatory (ISO) spectra of Klaas et al. (1997) show evidence for aromatic hydrocarbons (PAH) in emission. The present data do not have adequate spectral resolution to distinguish silicate absorption from an underlying silicate/blackbody/PAH continuum, although the observations of Smith et al. (1989) and Klaas et al. (1997) do suggest that PAH emission does contribute to the continuum that is being absorbed. The images where the northeast elongation clearly appears were taken at wavelengths where the very strong infrared emission features attributed to PAH emission are known to appear, i.e., 3.4, 7.8, 8.7, and $11.5 \mu \mathrm{m}$ in Arp 220 (3.3, 7.7, 8.6, and $11.3 \mu \mathrm{m}$ in the rest frame, Willner et al. 1977). It is possible that the extended structure in Arp 220 is traced most strongly in these features.

The interpretation of the $3.45-24.5 \mu \mathrm{m}$ observations depends critically on the size derived from the $24.5 \mu \mathrm{m}$ observations. The measured size of the western nucleus at $24.5 \mu \mathrm{m}, 0.73$, includes a significant component from the diffraction limit of the telescope (0".62). Subtracting 0".62 in quadrature from the observed size leads to a source size of 0 ".39. While the measured size of the PSF was consistently 0 ".62 during the observations reported here, and while we believe that the observations are nearly diffraction-limited, there is a possibility that seeing fluctuations could have increased the apparent size of the source. The seeing contribution, as extrapolated from the measured seeing at 12.5 $\mu \mathrm{m}$, could be as large as $0^{\prime \prime} .3$. If seeing contributed $0^{\prime \prime} .3$ to the image diameter at $24.5 \mu \mathrm{m}$, the source diameter is 0.25 . Thus a range of source diameters at $24.5 \mu \mathrm{m}$ of $0.25-0.39$ is possible.

The $24.5 \mu \mathrm{m}$ emission of the western source is approximately 3 times that of the eastern source. While the absorption is greater in the eastern source, the overall spectral energy distributions of the two nuclei do not suggest that the eastern source is at a significantly different temperature than the western one, and there is no strong evidence that the eastern source is contributing very differently to the total luminosity of the system than can be inferred directly from the observed $24.5 \mu \mathrm{m}$ emission. For simplicity we will therefore assume in the following discussion that the western and eastern nuclei have similar spectral energy distributions.

\subsection{Models}

The emission at wavelengths longer than $20 \mu \mathrm{m}$ can be modeled in several ways. In the first scenario we assume the largest source diameter, $0^{\prime \prime} .39$, and an optically thick nucleus that is unobscured at $24.5 \mu \mathrm{m}$. This size is just large enough to contain all of the VLBI radio point sources in the western nucleus (Smith et al. 1998), suggesting that this size is appropriate for the compact western nucleus. If the measured $24.5 \mu \mathrm{m}$ flux density in the western source is due to an optically thick source of diameter 0.39 , the $24.5 \mu \mathrm{m}$ brightness temperature is $85 \mathrm{~K}$. An $85 \mathrm{~K}$ blackbody of diameter 0 "'39 emits $365 \mathrm{mJy}$ at $1.3 \mathrm{~mm}$. The dust continuum emis- 
sion from the western source measured at $1.3 \mathrm{~mm}$ by Sakamoto et al. (1998) is $\sim 140 \mathrm{mJy}$, thus implying that the emissivity of the source is 0.5 at $1.3 \mathrm{~mm}$, consistent with it being optically thick at submillimeter wavelengths. If the eastern source has a spectral energy distribution similar to that of the western source, the two nuclei together account for essentially all of the flux density measured at $24.5 \mu \mathrm{m}$ in a 4" diameter beam or by IRAS at $25 \mu \mathrm{m}$ but for only $42 \mathrm{Jy}$ or $40 \%$ of the $104 \mathrm{Jy}$ observed at $60 \mu \mathrm{m}$ by IRAS (IRAS Explanatory Supplement 1988). Similarly, an extrapolation to the far-infrared implies that the nuclei together produce $\sim 5 \times 10^{11} L_{\odot}$, or $\sim 40 \%$ of the bolometric luminosity of Arp 220.

In this model, the remaining continuum flux at $60-100$ $\mu \mathrm{m}$ must come from the surrounding environment. This surrounding region cannot contribute significant flux at $24.5 \mu \mathrm{m}$ or $1.3 \mathrm{~mm}$, must be transparent at wavelengths shorter than $17.9 \mu \mathrm{m}$, and yet must contribute $\sim 60 \%$ of the bolometric luminosity of the system. This would be an extended starburst region of prodigious luminosity $\left(\sim 7 \times 10^{11} L_{\odot}\right)$. We will assume that $\lesssim 10 \%-15 \%$ of the emission at $24.5 \mu \mathrm{m}$ and $1.3 \mathrm{~mm}$, i.e., flux densities $\lesssim 1 \mathrm{Jy}$ and $\$ 30 \mathrm{mJy}$, respectively, would go undetected and could be in this component. We can fit the spectral energy distribution around $60-100 \mu \mathrm{m}$ with a source having a dust temperature of $40 \mathrm{~K}$ and an emissivity $\epsilon \sim v^{2}$, where $v$ is the frequency of the emission. To provide the excess flux of $\sim 70$ Jy at $100 \mu \mathrm{m}$, the source must be larger than $\sim 2^{\prime \prime}$ in diameter. This size is also consistent with the source size measured for Arp 220 at $350 \mu \mathrm{m}$ by D. Benford (1998, private communication). If the diameter of this source is taken as 2".6, or $\sim 900 \mathrm{pc}$, consistent with the observed size of the extended CO emission in the source (Scoville, Yun, \& Bryant 1997), the optical depth at $100 \mu \mathrm{m}$ is 0.7 . The steep emissivity law, $\epsilon \sim v^{2}$, is necessary to reduce the predicted dust continuum at $1.3 \mathrm{~mm}$ to levels that would not be detected in the existing maps (Sakamoto et al. 1998); the fit predicts a flux density of $30 \mathrm{mJy}$ for the $40 \mathrm{~K}$ component at $1.3 \mathrm{~mm}$. This emissivity behavior is physically reasonable based on a lack of resonances at wavelengths greater than $100 \mu \mathrm{m}$ in the grains producing the submillimeter continuum. If the $40 \mathrm{~K}$ component is distributed in a disklike structure, as will be discussed below, it can be nearly optically thick at $100 \mu \mathrm{m}$ while allowing more compact structure to be seen at shorter wavelengths. The low emission of a blackbody of this low temperature in the mid-infrared precludes using the present observations to define this component better. The large extent and luminosity of this component are also consistent with the distribution of the $\mathrm{CO}$ emission, which places $70 \%$ of the molecular gas outside the compact nuclei (Sakamoto et al. 1998), and with seeing both of the nuclei in absorption at $10 \mu \mathrm{m}$.

The apparent diffuse emission at $\lambda<17.9 \mu \mathrm{m}$ requires that dust significantly warmer than $85 \mathrm{~K}$ surrounds the compact nuclei. This dust is almost certainly heated externally to the compact nuclei, probably as a result of a large extended starburst environment. This provides a natural explanation of the low-level extended midinfrared emission associated with this region. It is reasonable that the excitation of the PAH emission seen in Arp 220 (Smith et al. 1989; Klaas et al. 1997; Genzel et al. 1998) is associated with the distributed starburst in the same way that similar features are seen in other starburst galaxies such as M82 (e.g., Willner et al. 1977). The observed 7.9 and $8.8 \mu$ m flux den- sities in Arp 220 from Table 2 imply that the $8 \mu \mathrm{m}$ luminosity is $\approx 4 \times 10^{10} L_{\odot}$. Under the simple assumption that $\lambda L_{\lambda} / L_{\text {bol }}$ in this extended starburst region is the same as that in M82 (Willner et al. 1977; Helou et al. 1998), we estimate a bolometric luminosity for this region of $1-4 \times 10^{11} L_{\odot}$ if the optical depth at $8 \mu \mathrm{m}$ is negligible. An optical depth at 8 $\mu \mathrm{m}$ of $\sim 0.5-1.5$ would then be consistent with the observed flux. Such an $8 \mu \mathrm{m}$ optical depth is consistent with the 10 $\mu \mathrm{m}$ optical depth of $\tau \sim 7$ for Arp 220 attributed to silicate absorption by Smith et al. (1989).

One possible problem with the extended starburst model is the faintness of the Brackett- $\alpha$ line. DePoy, Becklin, \& Geballe (1987) observed a faint extended line with a $5^{\prime \prime}$ diameter beam. When they corrected for a reddening of 50 mag of visual extinction, the strength of the line corresponded to a starburst luminosity of about $2-3 \times 10^{11} L_{\odot}$ (see Table 1 of DePoy et al.), which is substantially below the luminosity of $\sim 7 \times 10^{11} L_{\odot}$ discussed above. Further, the amount of extinction to an extended starburst region could be comparable to $A_{V}=50 \mathrm{mag}$ if $\tau_{100 \mu \mathrm{m}} \sim 0.7$. An $A_{V}$ of 50 mag is the extinction inferred from the silicate absorption feature corresponding to the total extinction in the central two sources.

The second scenario we present, which cannot be distinguished from the first on the basis of these observations, follows from the assumption that there is absorption at 24.5 $\mu \mathrm{m}$, so the intrinsic emission of the nuclei is attenuated by an opacity $\tau \approx 1-2$. The attenuating material is assumed to be in the proximity of the nuclei and is heated and reradiates as well, so that the compact sources provide the luminosity of Arp 220. In support of the assumption that $\tau \gtrsim 1$ at $24.5 \mu \mathrm{m}$ is the fact that there appears to be silicate absorption at $17.9 \mu \mathrm{m}$, most clearly visible in the eastern component (Fig. 2). In order for the compact nuclei to provide the total luminosity of Arp 220, the temperature of the western source must be between $102 \mathrm{~K}$, if the source diameter is 0.39 , and $128 \mathrm{~K}$, if the diameter of the source is 0 "' 25 . In the former case, the extinction to the source is $\tau=1.2$ at $24.5 \mu \mathrm{m}$; in the latter case it is $\tau=1.5$. The eastern nucleus has been assumed to have the same spectral energy distribution as the western nucleus and to suffer similar extinction. In order to match the observations at 1.3 $\mathrm{mm}$, the material surrounding the nuclei must become optically thin at that wavelength; the calculated values of emissivity are very similar to those proposed for the previous model. Since in this scenario the total bolometric luminosity is provided by the compact nuclei, the need for an extended (starburst) region is much diminished, but the long-wavelength radiation comes from the absorbing material in the neighborhood of the nucleus.

The overall energetics of the second model are shown in Figure 3; the gross appearance is much the same as for the first scenario discussed. The figure emphasizes the fact that however the luminosity is generated, the $24.5 \mu \mathrm{m}$ observations require a component at a temperature of $30-50 \mathrm{~K}$ and a size measured in arcseconds to radiate some significant fraction of the luminosity of Arp 220. Since both models are carefully contrived to fit the present data, we cannot distinguish between the two models on the basis of the available observations. The correct picture is probably a combination of the two pictures described above that involves some extinction at $24.5 \mu \mathrm{m}$ and some starburst activity extended on a $2^{\prime \prime}-3$ " scale, providing some of the luminosity of Arp 220. 


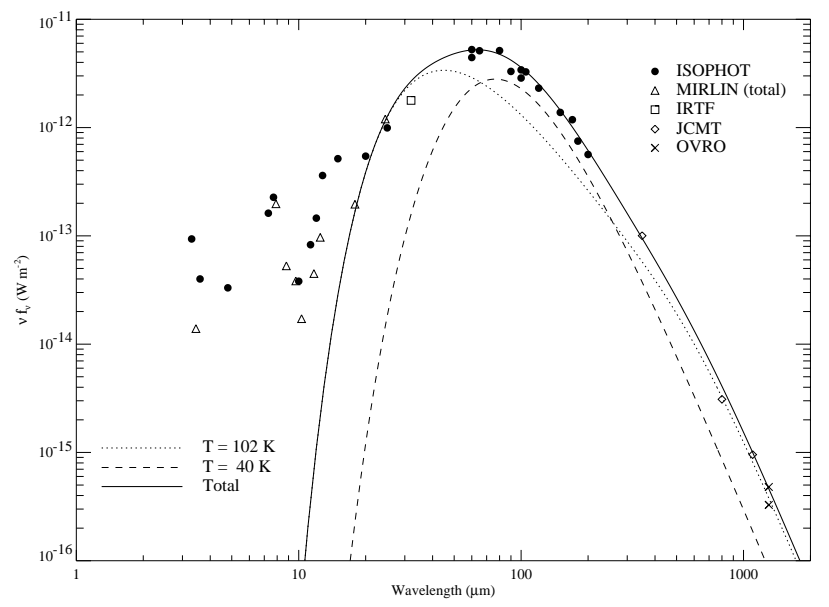

FIG. 3.-Various observations of Arp 220 are shown together with the second model of obscured nuclei presented in the text. The data points include the present observations with a 4" diameter beam (MIRLIN), the photometry from Klaas et al. (1997) (ISOPHOT), the $32 \mu \mathrm{m}$ photometry from Wynn-Williams \& Becklin (1993) (IRTF), submillimeter data from Rigopoulou, Lawrence, \& Rowan-Robinson (1996) (JCMT), and millimeter data from Sakamoto et al. (1998) (OVRO). For the OVRO data, both the total and the western nuclear fluxes are plotted by crosses. For the model, plotted as a solid line, we assume that almost all the luminosity of Arp 220 comes from two compact nuclei and that the total luminosity of the galaxy is 1.3 times that of the western nucleus, the ratio being determined from the $24.5 \mu \mathrm{m}$ data. A blackbody temperature of $102 \mathrm{~K}$ was derived for the western nucleus with the assumptions that the diameter of the nucleus is 0.39 and that $v f_{v}$ at the peak of this blackbody curve is $77 \%$ of $v f_{v}$ at $60 \mu \mathrm{m}$ calculated from the IRAS data. A foreground extinction was applied in the form $\exp \left[-\tau_{24.5 \mu \mathrm{m}}\left(v / v_{24.5 \mu \mathrm{m}}\right)^{2}\right]$ with $\tau_{24.5 \mu \mathrm{m}}=1.2$, which gives a good fit to the western nucleus data points at 17.9 and $24.5 \mu \mathrm{m}$ (not plotted). This extinction-applied curve was then multiplied by 1.3 to take into account the contribution from the eastern component, which was assumed to have the same spectral energy distribution. Finally, the result was multiplied by $\left\{1-\exp \left[-(v / 0.43 \mathrm{TH} z)^{2}\right]\right\}$ to make the emitting region optically thin at the longer wavelengths so that the curve matches the $1.3 \mathrm{~mm}$ OVRO data. The result is plotted as the dotted line. To obtain the final fit (solid line), emission from another lower temperature dust component in the form of $B_{v}(T=40 \mathrm{~K})\left\{1-\exp \left[-(v / 3 \mathrm{TH} z)^{2}\right]\right\}$ was added (dashed line). The size of the $40 \mathrm{~K}$ emission was assumed to be 46 square arcsec, which was derived by equating the energy emitted by the 40 $\mathrm{K}$ component with the energy absorbed by the screen in front of the $102 \mathrm{~K}$ blackbody nuclei.

\subsection{General Discussion}

Independently of the details of the models, the observations of compact sources at $24.5 \mu \mathrm{m}$ and $1.3 \mathrm{~mm}$ and the inference that even at $100 \mu \mathrm{m}$ the sources must have substantial optical depth and comparatively small size lead to the conclusion that we are seeing a wide range of source temperatures and sizes with sources that are optically thick at all observed wavelengths shorter than $25 \mu \mathrm{m}$. The nuclear disks inferred from the millimeter $\mathrm{CO}$ observations of Sakamoto et al. (1998) and Downes \& Solomon (1998) present a natural way to accommodate these observations as well as the global feature from Figure $1 a$ that the images at all wavelengths from 3.45 to $24.5 \mu \mathrm{m}$ show the same qualitative structure.

If the east and west sources are both disks viewed such that both are reasonably close to the plane of the sky (i.e., significantly away from edge-on), the apparent global morphology will be the same at all wavelengths, the size will increase with wavelength, and the source can appear to have significant optical depth at each wavelength. If the disks are warped and thick, the outer disk could provide the apparent cold silicate absorption through which the hotter material is seen. Thus we conclude that the infrared observations of Arp 220 are entirely consistent with the tilted disks suggested from the millimeter $\mathrm{CO}$ data.

The observed emission at wavelengths smaller than 17.9 $\mu \mathrm{m}$ is well in excess of that predicted for either of the scenarios described above. Specifically, at $12.5 \mu \mathrm{m}$ an $85 \mathrm{~K}$ blackbody of 0.39 diameter emits $75 \mathrm{mJy}$, compared to the observed emission of $269 \mathrm{mJy}$, while if the attenuation keeps increasing in proportion to $v^{2}$, the emission from an attenuated $100-130 \mathrm{~K}$ blackbody is less than $1 \mathrm{mJy}$. Because dust opacity increases to shorter wavelengths, this cannot be a result of seeing deeper into a uniform distribution of material. Undoubtedly, the nuclear region is geometrically complex and must include nonuniform extinction over both nuclei. The disks seen by Sakamoto et al. (1998) provide a natural means of allowing visibility into regions that appear optically thick at long wavelengths while allowing radiation at shorter wavelengths to escape.

In any viable model, the luminosity of Arp 220, or a significant fraction of it, is generated in a volume $\sim 10^{6} \mathrm{pc}^{3}$, with an energy output per unit volume $\gtrsim 100$ times that in the extended nuclear starburst in M82. No direct observational evidence has been presented for a nonstellar origin of the luminosity originating in the nuclei of Arp 220. Highexcitation infrared fine-structure lines such as [O IV] $24 \mu \mathrm{m}$ and [Ne v] $14 \mu \mathrm{m}$ have not been detected in Arp 220 in recent observations with the ISO Short Wavelength Spectrometer (SWS) (Genzel et al. 1998). The present results show that the nuclei in Arp 220 are probably optically thick even at $24.5 \mu \mathrm{m}$, and therefore the existence of a central AGN in these nuclei cannot be probed even with these mid-infrared lines.

\section{SUMMARY AND CONCLUSIONS}

High spatial resolution imaging of the nuclear regions of Arp 220 from 3.45 to $24.5 \mu \mathrm{m}$ has shown that the central source consists of at least two compact sources with diffuse emission surrounding them. Both compact sources are heavily obscured at $10 \mu \mathrm{m}$ owing to silicate absorption. The western nucleus is marginally spatially resolved and provides, depending on the detailed structure, at least $30 \%-70 \%$ of the bolometric luminosity of Arp 220 . The source that generates this luminosity must have a luminosity density $\gtrsim 100$ times that of the starburst region in M82. The optical depth inferred for this source implies that there is likely no meaningful probe of the power source short of radio wavelengths. The large number of supernova remnants reported by Smith et al. (1998) clearly demonstrates that young stars must be contributing significantly to the luminosity of this source. The eastern nucleus appears to contribute about one-third of the luminosity of the western nucleus and appears to be spatially extended in the northeast direction.

Despite the fact that there is significant extinction of 3.45-24.5 $\mu \mathrm{m}$ radiation in Arp 220, the presence of emission at $3.45-12.5 \mu \mathrm{m}$ indicates that there are lines of sight to hotter regions relatively free of extinction. The two models we have proposed, one in which the majority of the total luminosity is produced in an extended star formation region and another in which the majority is produced in two compact but extincted regions, both allow a geometry in which short-wavelength emission can escape. Disks of 
material with the range of temperatures described here that are inclined to our line of sight provide a natural mechanism for achieving this.

If the extinction to the western source at $24.5 \mu \mathrm{m}$ is small, the western source has a brightness temperature at $24.5 \mu \mathrm{m}$ of $85 \mathrm{~K}$; if the extinction is actually higher, it will be hotter. Both models must therefore include hot $(T>85 \mathrm{~K})$ embedded sources, and hence the radiative transfer in both implies that the colder surrounding material, which will emit most strongly at wavelengths greater than $60 \mu \mathrm{m}$, must be extended. If most of the luminosity is generated in the compact sources, this extended emission will have a size $\gtrsim 1^{\prime \prime}$ in radius, whereas if most of the luminosity is generated outside the nuclei, this colder extended region must be at least $50 \%$ larger.

Both of these models can reproduce the bolometric luminosity of the galaxy if the flux is extrapolated to $60-100 \mu \mathrm{m}$ where the bulk of the luminosity of Arp 220 is observed.
Both are also consistent with the measured sizes of the nuclear region in $\mathrm{CO}$ and continuum dust emission.

B. T. S., G. N., and K. M. are supported by grants from NASA and NSF. We thank J. Aycock, R. Goodrich, R. Moskitis, and the entire Keck staff for their help establishing the visitor port and obtaining these observations, and R. Chary, A. Evans, D. Koerner, K. Sakamoto, D. Sanders, and N. Scoville for helpful discussions about Arp 220 and these observations. The W. M. Keck Observatory is operated as a scientific partnership between the California Institute of Technology, the University of California, and the National Aeronautics and Space Administration. It was made possible by the generous financial support of the W. M. Keck Foundation. This research has made use of the NASA/IPAC Extragalactic Database, which is operated by the Jet Propulsion Laboratory, Caltech, under contract with NASA.
Arp, H. C. 1966, ApJS, 14, 1

Baan, W. A., \& Haschick, A. D. 1995, ApJ, 454, 745

Condon, J. J., Huang, Z. P., Yin, Q. F., \& Thuan, T. X. 1991, ApJ, 378, 65

DePoy, D. L., Becklin, E. E., \& Geballe, T. R. 1987, ApJ, 316, L63

Downes, D., \& Solomon, P. M. 1998, ApJ, 507, 615

Genzel, R., et al. 1998, ApJ, 498, 579

Graham, J., R., Carico, D. P., Matthews, K., Neugebauer, G., Soifer, B. T., \& Wilson, T. D. 1990, ApJ, 354, L5

Helou, G., Lu, N. Y., Werner, M., Malhotra, S., \& Silberman, N. 1998, ApJL, submitted

IRAS Catalogs and Atlases: Explanatory Supplement. 1988, ed. C. A. Beichaman, G. Neugebuaer, H. J. Habing, P. E. Clegg, \& T. J. Chester (Washington, DC: GPO)

Keto, E., Ball, R., Arens, J., Jernigan, G., \& Meixner, M. 1992, ApJ, 389, 223

Klaas, U., Haas, M., Heinrichsen, I., \& Schulz, B. 1997, A\&A, 325, L21

Larkin, J. E., Armus, L., Knop, R. A., Matthews, K., \& Soifer, B. T. 1995, ApJ, 452, 599

Miles, J. W., Houck, J. R., Hayward, T. L., \& Ashby, M. L. N. 1996, ApJ, 465, 191

Ressler, M. E., Werner, M. W., Van Cleve, J., \& Choa, H. 1994, Exp. Astron., 3, 277

\section{REFERENCES}

Rigopoulou, D., Lawrence, A., \& Rowan-Robinson, M. 1996, MNRAS, 278,1049

Sakamoto, K., Scoville, N. Z., Yun, M. S., Crosas, M., Genzel, R., \& Tacconi, L. J. 1998, ApJ, submitted

Sanders, D. B., Soifer, B. T., Elias, J., Madore, B., Matthews, K., Neugebauer, G., \& Scoville, N. 1988, ApJ, 325, 74

Scoville, N. Z., et al. 1998, ApJ, 492, L107

Scoville, N. Z., Yun, M. S., \& Bryant, P. M. 1997, ApJ, 484, 702

Smith, C. H., Aitken, D., K., \& Roche, P., F. 1989, MNRAS, 241, 425

Smith, H. E., Lonsdale, C. J., Lonsdale, C. J., \& Diamond, P. J. 1998, ApJ, 493, L17

Soifer, B. T., et al. 1984, ApJ, 283, L1

Soifer, B. T., Sanders, D. B., Madore, B. F., Neugebauer, G., Danielson, G. E., Elias, J. H., Lonsdale, C. J., \& Rice, W. L. 1987, ApJ, 320, 238

Toomre, A., \& Toomre, J. 1972, ApJ, 178, 623

van der Werf, P. P. 1998, Proc. KNAW Colloq., ed. H. J. A. Rottgering,

P. N. Best, \& M.D. Lehnert (Dordrecht: Kluwer )

Willner, S. P., Soifer, B. T., Russell, R. W., Joyce, R. R., \& Gillett, F. C. 1977, ApJ, 217, L121

Wynn-Williams, C. G., \& Becklin, E. E. 1993, ApJ, 412, 535 